\title{
ARTICLE \\ Pharmacokinetics trumps pharmacodynamics during cocaine choice: a reconciliation with the dopamine hypothesis
}

\section{of addiction}

\author{
Ludivine Canchy $^{1,2}$, Paul Girardeau ${ }^{3}$, Audrey Durand ${ }^{4}$, Caroline Vouillac-Mendoza ${ }^{1,2}$ and Serge H. Ahmed ${ }^{1,2}$
}

\begin{abstract}
Cocaine is known to increase brain dopamine at supranormal levels in comparison to alternative nondrug rewards. According to the dopamine hypothesis of addiction, this abnormally large dopamine response would explain why cocaine use is initially highly rewarding and addictive. Though resting on solid neuroscientific foundations, this hypothesis has nevertheless proven difficult to reconcile with research on cocaine choice in experimental animals. When facing a choice between an intravenous bolus of cocaine and a nondrug alternative (e.g., sweet water), both delivered immediately after choice, rats do not choose the drug, as would be predicted, but instead develop a strong preference for the nondrug alternative. Here we report evidence that reconciles this finding with the dopamine hypothesis of addiction. First, a systematic literature analysis revealed that the delays of effects of intravenous cocaine on nucleus accumbens dopamine are of the order of tens of seconds and are considerably longer than those of nondrug reward. Second, this was confirmed by measuring response times to cocaine omission during self-administration as a behavioral proxy of these delays. Finally, when the influence of the drug delays was reduced during choice by adding an increasing delay to both the drug and nondrug rewards, rats shifted their choice to cocaine. Overall, this study suggests that cocaine is indeed supranormal in reward magnitude, as postulated by the dopamine hypothesis of addiction, but is less preferred during choice because its pharmacokinetics makes it an inherently more delayed reward than the alternative. Reframing previous drug choice studies in rats as intertemporal choice studies reveals that the discounting effects of delays spare no rewards, including supranormal ones, and that during choice, pharmacokinetics trumps pharmacodynamics.
\end{abstract}

Neuropsychopharmacology (2021) 46:288-296; https://doi.org/10.1038/s41386-020-0786-9

\section{INTRODUCTION}

Cocaine is known to increase ventral striatal dopamine at supranormal levels in comparison to alternative nondrug rewards, such as, for instance, food or social reward [1-4]. According to the dopamine hypothesis of addiction, this abnormally large dopamine response would explain why cocaine is initially highly rewarding and addictive [1-7]. It should also make cocaine a more attractive and preferred reward in comparison to alternative nondrug rewards. Though the dopamine hypothesis of addiction rests on solid neuroscientific foundations, the latter prediction has proven difficult to reconcile with research on drug choices in experimental animals that seems to show the opposite [8, 9]. When facing a concurrent choice between taking an immediate intravenous bolus of cocaine and having immediate access to a nondrug alternative (e.g., sweet water), rats do not choose the drug, but instead develop a strong preference for the nondrug alternative, sometimes to the complete exclusion of continued drug use [10-18]. This preference has now been observed with many drugs of abuse, including heroin and methamphetamine [12, 18-23], with different kinds of nondrug options [11, 20, 24-26] and also following different drug and/or behavioral histories $[10-12,18,20,26]$.
Though this discrepancy cries out for an explanation, it has so far been largely overlooked. Recently, we proposed to resolve this discrepancy by considering some of the inherent differences that exist between drug and nondrug options, particularly in reward delays $[8,9]$. Though every effort was made in previous studies to equalize the parameters of choice between drug and nondrug rewards (e.g., same effort; same probability), it has remained particularly difficult to equalize reward delays. Specifically, though the drug and nondrug options are both delivered immediately after a choice, pharmacokinetics prevents the drug from producing its rewarding effects immediately [27-29], even when rapidly infused by the intravenous route, as was the case in previous drug choice studies (i.e., typically within less than $5 \mathrm{~s}$ ). In fact, even if the drug was delivered instantaneously to the venous blood stream, there would remain the incompressible delays of drug distribution, diffusion and action into the brain [27,30]. Thus, it is likely that cocaine reward is in fact more delayed than the nondrug reward, though by how much exactly is currently uncertain (see below).

Differences in delays between different options can exert profound influence on choice outcomes in humans and other animals [31-34]. In particular, rats are highly sensitive to reward delays; they typically prefer a small reward now over a larger reward

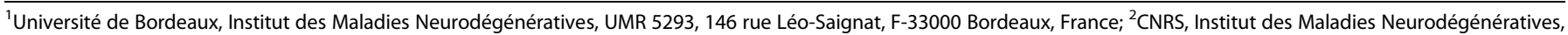

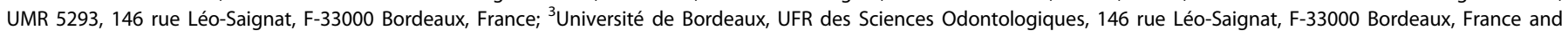
${ }^{4}$ Imetronic $^{\circledR}, 156$ avenue Jean Jaurès, F-33600 Pessac, France

Correspondence: Serge H. Ahmed (serge.ahmed@u-bordeaux.fr)

Received: 5 June 2020 Revised: 10 July 2020 Accepted: 24 July 2020

Published online: 30 July 2020 
that is delayed by only few seconds [35-41]. For instance, when hungry rats are facing a choice between one food pellet delivered immediately and four food pellets delivered after a delay of $20 \mathrm{~s}$, they typically choose the smaller amount of food, ending up eating less [35]. Regardless of the mechanisms underlying these delay effects, the pharmacokinetic delays associated with cocaine reward, if sufficiently long, could thus explain rats' preference for the nondrug alternative in previous drug choice studies, without challenging the dopamine hypothesis of addiction [8,9]. Reframing drug choice studies as intertemporal choice studies between a delayed drug reward and an immediate nondrug alternative may reveal that the known discounting effects of delays spare no rewards, including supranormal ones, in rats.

One way to test this delayed drug reward hypothesis could be to try to equalize the delay of the two options by increasing the delay to the nondrug reward, the inverse (i.e., reducing the delay to drug reward to make it quasi-immediate) being not feasible (see also Discussion). For instance, previous research has shown that increasing the delay to the nondrug option can increase drug choice [11] and eventually shift preference toward the drug $[26,42,43]$. However, without precise knowledge of the delay to drug reward, this approach remains inconclusive because we do not know when the delay added to the nondrug option becomes equal to the unknown delay to drug reward. Thus, knowing the delay to drug reward seems a prerequisite for the validity of this approach. However, as it will soon become apparent, addressing this question is not as simple as it may appear. For comparison, the delay to a nondrug reward is the time interval between a choice response and reward consumption, two observable behavioral events. For instance, with the sweet water option used in our previous choice studies, it is the time interval between the operant response and the onset of sweet water consumption in an adjacent drinking cup [11]. Of particular note, when there is no programmed delay, this time interval is close, but not equal to 0 , because it takes some time to move between the response location and the adjacent reward location (i.e., typically within 1-2 s) [11].

It is not easy to apply this behavioral definition to intravenous cocaine or to any other intravenous drugs of abuse for that matter. Though we know precisely when an intravenous drug infusion begins and ends (and the interval between the two is usually of few seconds), we do not know when exactly drug reward begins $[8,9,44,45]$. We only know from pharmacokinetics that it cannot start with the drug infusion. After a rat has made a response that triggers an immediate drug infusion, there is no observable behavior that could mark or report unambiguously the onset of the drug reward experience. We previously reported that it takes about $6 \mathrm{~s}$ to observe an overt behavioral reaction following the onset of an intravenous cocaine infusion [11], but we do not know if this reaction precedes, coincides with or even follows the onset of drug reward. As will be explained later, there may be other indirect ways to estimate this delay, but each has its own problems. Thus, the exact delay of onset of cocaine reward remains largely an unknown.

We thus need an approach to test the delayed drug reward hypothesis without prior precise knowledge of the delay to cocaine reward. Previous research has shown that when a sufficiently long delay is added both to a small, immediate reward and to a larger, delayed reward, rats change their preference in favor of the larger reward, as if its longer delay no longer mattered [34]. For instance, in one paradigmatic experiment, a rat that preferred two food pellets immediately over four pellets delayed by $20 \mathrm{~s}$ reversed its preference to the larger reward when a delay of $15 \mathrm{~s}$ was added to the two options [46]. Importantly, this change occurred despite the fact that the larger reward was still more delayed by $20 \mathrm{~s}$ than the smaller one (i.e., the rat now preferred four pellets after $35 \mathrm{~s}$ over two pellets after $15 \mathrm{~s}$ ) [46]. This preference reversal phenomenon has been observed in pigeons [47-49], in rats [39, 46, 50,51], in monkeys [52] and in humans [53, 54], and is thought to be a consequence of hyperbolic delay discounting [31, 53], though other plausible explanations exist $[33,55,56]$. Regardless of the underlying mechanisms, however, the preference reversal phenomenon suggests a unique way to test the delayed drug reward hypothesis. Thus, if cocaine reward is really larger in magnitude than sweet water reward, but is not chosen because it is more delayed, then adding a sufficiently long programmed delay to these two options should cause rats to shift their choice from sweet water to cocaine, even if the latter remains more delayed. If instead cocaine reward is not larger in magnitude than sweet water reward, then no preference reversal should be observed.

The goal of the present study was to test this unique prediction. We first conducted a systematic analysis of the literature to try to obtain more precise information on the delay of action of intravenous cocaine on ventral striatal dopamine parameters (i.e., dopamine uptake inhibition and extracellular concentrations) (see Supplemental Online Information). Though there is a debate on the exact behavioral significance of these parameters, they are arguably one of the earliest markers of cocaine reward in the brain $[30,45,57]$. This analysis revealed that the delays of action of intravenous cocaine on ventral striatal dopamine are of the order of tens of seconds, as was suggested previously [30], and are considerably longer than those measured in response to food reward (Tables S1 and S2, Figs. S1-S3). We complemented this research with a behavioral measurement approach. We reasoned that rats should learn about the delay to cocaine reward during cocaine self-administration. Therefore, their response time to a drug omission should be a reflection, albeit an indirect one, of this delay. Finally, we conducted a preference reversal experiment, as outlined above. Overall, our findings confirm the delayed drug reward hypothesis and reconcile previous drug choice studies with the dopamine hypothesis of addiction. There is little doubt that cocaine reward is supranormal in magnitude, but its inherently long delay makes it a less preferred option during choice.

\section{METHODS}

Subjects

A total of 83 adult male Wistar rats $(225-250 \mathrm{~g}$ at the beginning of experiments, Charles River, Lyon, France) were used. Rats were housed in groups of two and were maintained in a light- (reverse light-dark cycle), humidity- $(60 \pm 20 \%)$, and temperature-controlled vivarium $\left(21 \pm 2^{\circ} \mathrm{C}\right)$. All behavioral testing occurred during the dark phase of the light-dark cycle. Food and water were freely available in the home cages throughout the duration of the experiment. Home cages were enriched with a nylon gnawing bone and a cardboard tunnel (Plexx BV, The Netherlands). 28 rats did not complete the behavioral experiments which lasted several months, thereby leaving a total of 55 rats for final analysis. Rats did not complete the experiments due to a variety of factors (e.g., failure to acquire cocaine self-administration; infection; catheter failure).

All experiments were carried out in accordance with institutional and international standards of care and use of laboratory animals [UK Animals (Scientific Procedures) Act, 1986; and associated guidelines; the European Communities Council Directive (2010/63/UE, 22 September 2010) and the French Directives concerning the use of laboratory animals (décret 2013-118, 1 February 2013)]. The animal facility has been approved by the Committee of the Veterinary Services Gironde, agreement number A33-063-922.

Standard experimental procedures are described in the Supplemental Online Material. The timeline of the different phases of the behavioral experiments is shown in Fig. 1.

Specific experimental procedures

Response times to drug reward omissions. The rationale behind this test is based on well-established prior knowledge on 
A

Experimental timeline of drug omission experiment

\begin{tabular}{|c|c|c|c|}
\hline $\begin{array}{c}\text { Habituation to } \\
\text { operant box } \\
\text { (2 sessions })\end{array}$ & $\begin{array}{c}\text { Cocaine SA } \\
\text { Initial training } \\
\text { (16 sessions })\end{array}$ & $\begin{array}{c}\text { Cocaine SA } \\
\text { TO cue off } \\
(10 \text { sessions })\end{array}$ & $\begin{array}{c}\text { Dose omission } \\
\text { testing } \\
(18 \text { sessions })\end{array}$ \\
\hline
\end{tabular}

$\mathrm{B}$

Experimental timeline of preference reversal experiment

\begin{tabular}{|c|c|c|c|c|}
\hline $\begin{array}{l}\text { Habituation to } \\
\text { operant box } \\
\text { (2 sessions) }\end{array}$ & $\begin{array}{l}\text { Cocaine SA } \\
\text { Initial training } \\
\text { (19 sessions) }\end{array}$ & $\begin{array}{c}\text { Alternate training } \\
\text { Cocaine/Sucrose } \\
\text { (6 sessions) }\end{array}$ & $\begin{array}{l}\text { Discrete choice } \\
\text { training } \\
\text { (12 sessions) }\end{array}$ & $\begin{array}{l}\text { Programmed } \\
\text { delay testing } \\
\text { (35 sessions) }\end{array}$ \\
\hline
\end{tabular}

Fig. 1 Experimental timeline. a Timeline of the successive phases of the response omission time experiment with the corresponding number of sessions. b Timeline of the successive phases of the preference reversal experiment with the corresponding number of sessions. SA self-administration.

intravenous cocaine self-administration under an FR1 schedule of reinforcement [58-61]. Briefly, during acquisition of cocaine selfadministration, rats learn that each response is rewarded by cocaine. They then use this information to regulate their intake of cocaine, probably to maintain its rewarding effects around some preferred level and also to avoid overdosing. Typically, after acquisition, they begin a session by responding at a rapid rate to load on cocaine. This initial, brief loading period is then followed by a maintenance period during which rats respond at regular intervals until the end of the session. The length of these regular intervals depends on the unit dose of cocaine available for selfadministration: the larger the dose, the longer the inter-response interval. For instance, at the dose used here during training (i.e., $0.25 \mathrm{mg}$ per injection), this interval lasts on average about $280 \mathrm{~s}$ in our experimental conditions. Importantly, in our experimental conditions, we typically find that during the maintenance period, rats rarely respond during the 20 -s time-out period that immediately follows the rewarded response, a behavior that is also observed when the time-out period is not signaled. In this experiment, rats emitted $0.21 \pm 0.04$ time-out response per rewarded response when the time-out period was signaled and $0.29 \pm 0.06$ when it was not signaled $[F(1,16=2.17, N S)]$.

The relative absence of responding during the post-response time-out period could suggest that after completion of FR1 responding, rats experience quasi-immediately cocaine reward and/or some conditioned interoceptive drug cues. Alternatively and more in accordance with the present hypothesis, it could also indicate that rats have learned to expect that cocaine reward will only occur after a long post-response delay. One way to disentangle these two possibilities is to measure how fast rats respond after drug reward omissions during the maintenance phase of cocaine self-administration. In theory, if responding were followed immediately by cocaine reward and/or some conditioned interoceptive drug cues, then rats should rapidly detect the drug reward omission and thus respond again rapidly (i.e., within few seconds). In contrast, if they have learned that cocaine reward is associated with a long delay, then they should wait a period of time that is at least as long as the drug reward delay before responding again after a drug omission. Since it should take some time to become confident that a drug reward omission has occurred, the omission response time should be slightly longer than the real delay to cocaine reward.

To measure the omission response time, rats were initially trained to self-administer cocaine $(0.25 \mathrm{mg}$ per injection) during 16 sessions, as described above. Then, starting from session 16 onward, the time-out cue was removed. This was done to avoid any external influence on responding. As expected, removal of the time-out cue had no significant impact on the maintenance of cocaine self-administration (Fig. S4). Rats were then tested without the time-out cue during seven additional sessions of cocaine self-administration before sessions with drug reward omissions began. These sessions were identical to preceding training sessions of cocaine self-administration, except that cocaine reward was occasionally omitted after some FR1 responses during the maintenance period, forcing rats to respond a second time to obtain it. The omission response time was the interval between these two consecutive responses. Drug reward omissions were unsignaled and occurred aperiodically after every 5-7 rewarded responses. No drug reward omissions occurred during the loading period (i.e., first five injections). The number of drug reward omissions was initially set to 5 per 3 -h session (i.e., first four warming-up sessions). Subsequently, to obtain more measurements of omission response times per session, the daily session length was increased to 4.5 -h which allowed to test eight drug reward omissions. Rats were tested under this condition during 10 consecutive sessions, amounting to a maximum of 80 measurements of omission response time per individual rat. Finally, at the end of the experiment, the unit dose of cocaine was halved (i.e., $0.125 \mathrm{mg}$ instead of $0.25 \mathrm{mg}$ ) to double the number of injections per session and thus the number of drug reward omissions tested per session, from 8 to 16 . Rats were tested with this dose during four consecutive sessions, which allowed a maximum of 64 measurements of omission response time per individual rat. Since a change of dose affects the amount of, but not the delay to cocaine reward, this test was also used to further validate the drug reward omission procedure.

\section{Preference reversal experiment}

Alternate operant training. Rats were first trained to selfadminister cocaine during 19 daily sessions, as described above. Starting from session 20 onward, they were also trained on alternate days to respond for sweet water by pressing on a different lever. In total, there were three sweet water training sessions alternating with three sessions of cocaine selfadministration before choice testing [10, 11, 62, 63]. On sweet water sessions, the lever not previously associated with cocaine (i.e., lever S) was extended to mark the onset of the session and to signal sweet water availability; the lever previously associated with cocaine (i.e., lever $C$ ) remained retracted. Responding on lever $\mathrm{S}$ was rewarded by a $20-\mathrm{s}$ access to water sweetened with $10 \%$ sucrose delivered in the adjacent drinking cup on the same wall and was signaled by illumination of the cue-light above lever $\mathrm{S}$ for $20 \mathrm{~s}$. Specifically, each FR1 response triggered the immediate activation of the sucrose syringe pump which filled the drinking cup with $0.08 \mathrm{ml}$ of sweet water within $3 \mathrm{~s}$. Additional volumes could be obtained during the remaining $17 \mathrm{~s}$ by contacting the cup (i.e., one volume every $2.8 \mathrm{~s}$ ). The maximum volume that a rat could drink per 20-s access period was $0.32 \mathrm{ml}$ of sweet water and thus $9.6 \mathrm{ml}$ per session (i.e., $30 \times 0.32 \mathrm{ml}$ ). We used the same conditions of access to sweet water as in our previous choice experiments $[10,11,62,63]$. The session ended after rats had earned a maximum of 30 sweet water rewards which typically occurred in less than $30 \mathrm{~min}$. The data obtained during alternate operant training sessions (i.e., three cocaine sessions, three sucrose sessions) are presented in Fig. S5.

Discrete-trials choice procedure. After acquisition of lever pressing for cocaine and sweet water, rats were allowed to choose during several consecutive daily sessions between lever $C$ and lever $S$ on a discrete-trials choice procedure. Each daily choice session consisted of 16 discrete trials, spaced by $10 \mathrm{~min}$, and divided into two successive phases, sampling (4 trials) and choice (12 trials). During sampling, each trial began with the presentation of one single lever in this alternate order: $C-S-C-S$. Lever $C$ was presented first to prevent an eventual drug-induced taste aversion conditioning or negative affective contrast effects [11]. If rats responded on the available lever within $5 \mathrm{~min}$, this triggered the 
immediate retraction of the lever, the immediate activation of the relevant syringe pump (i.e., sucrose or cocaine as described above) and the immediate illumination of the cue-light above the sampled lever for 20-s. If rats failed to respond within $5 \mathrm{~min}$, the lever retracted until the next trial. Thus, during sampling, rats were allowed to evaluate each option separately before making their choice. Choice trials were identical to sampling trials, except that they began with the presentation of both levers $S$ and $C$ and ended with their simultaneous retraction. Rats had to respond on one of the two levers to make their choice and obtain the corresponding reward. During sampling and choice, the response requirement was set to two consecutive responses to avoid eventual accidental choice. A response on the alternate lever before satisfaction of the response requirement reset it. Response resetting occurred very rarely, however. Rats were tested in this discrete-trials choice procedure during 12 daily sessions before being tested with different programmed delays.

Adding a programmed delay to both options. After stabilization of preference, the same programmed delay was added to both options using a choice procedure identical to that described above. Thus, when rats responded during sampling or choice, this triggered the immediate retraction of the lever(s) and the immediate illumination of the relevant cue-light for $20 \mathrm{~s}$ (+ programmed delay), but the relevant syringe pump (i.e., cocaine or sucrose) was activated only after a programmed delay. Four programmed delays were tested in the following ascending order: 10, 20, 40, and $60 \mathrm{~s}$. Each delay was tested during at least six consecutive daily sessions until stabilization of groupaverage behavior (i.e., no increasing or decreasing trend across three consecutive sessions). In total, rats were tested during 35 consecutive sessions. The last three baseline choice sessions preceding testing with programmed delays served as the $0-5$ delay control condition.

Data analysis. All data were subjected to relevant repeated measures ANOVAs, followed by Tukey post hoc tests where relevant. Comparisons with a fixed theoretical level (e.g., 50\%) were conducted using one sample $t$-tests. Alpha levels for detecting statistical significant differences were set to $p<0.05$. Statistical analyses were run using Statistica, version 7.1 (Statsoft Inc., Maisons-Alfort, France).

\section{RESULTS}

Omission response times during cocaine self-administration We analyzed for each individual rat the distribution of omission response times as a function of the cocaine dose available (i.e., a maximum of 80 and 64 measured times at 0.25 and $0.125 \mathrm{mg}$, respectively) (Fig. 2a). Overall, for all individuals, most omission response times were relatively long and variable (CVs $>50 \%)$, but they did not change significantly with the dose available ( $p$ values $>0.05$ ). Individual mean omission response times ranged from 24.0 to $88.1 \mathrm{~s}$ at the dose of $0.25 \mathrm{mg}$ and from 22.6 to 113.2 at the dose of $0.125 \mathrm{mg}$. When all individual mean data were pooled, the mean omission response time of the group was $44.5 \pm 16.1 \mathrm{~s}$ (mean \pm 1 standard deviation) for the dose of $0.25 \mathrm{mg}$ and $43.5 \pm 22.8 \mathrm{~s}$ for the dose of $0.125 \mathrm{mg}$ (Fig. 2b). This difference was not statistically significant $(\mathrm{F} 1,16=1.89, \mathrm{NS})$. The shortest delays that were statistically indistinguishable to these two measured delays were $37 \mathrm{~s}[\mathrm{t}(16)=1.91, p>0.05]$ and $32 \mathrm{~s}[\mathrm{t}(16)=2.08, p>0.05]$, respectively. Overall these omission response times are within the interval range defined by the delays $\mathrm{D} 10 \%$ and $\mathrm{D} 100 \%$ of the effects of intravenous cocaine on ventral striatal dopamine parameters (Fig. S3). For comparison, the mean inter-response interval during the three baseline sessions of cocaine self-administration preceding sessions of drug reward omissions was about 10 times longer: $277.4 \pm 114.4$ s (mean \pm 1 standard deviation).
If the omission response time is a genuine reflection of the delay to cocaine reward, as hypothesized here, then it should increase after adding a programmed delay between the response and the drug infusion during cocaine selfadministration. To test this prediction, we measured in a separate group of rats $(n=16)$ how different programmed delays influenced omission response times (six drug reward omissions per 3.5 -h session). Three programmed responseinfusion delays were tested $(0,20$, and $10 \mathrm{~s}$, in that order), each during at least seven consecutive sessions. Only the results obtained during the last five sessions of each condition were considered for analysis. Rats used in this study were initially trained to self-administer cocaine $(0.25 \mathrm{mg}$ per injection over $2 \mathrm{~s})$ using a modified FR1 schedule. Briefly, immediately after each response, the lever was retracted for $30 \mathrm{~s}$. Since most rats did not respond before that time after a drug omission, as shown above, this modification should be of little consequence on omission response times. This was indeed the case. When no programmed delay was added between the response and the drug infusion, individual mean omission response times were comparable to those measured in the previous experiment and ranged from 34.9 to $106.9 \mathrm{~s}$ (Fig. 3a). In addition, the mean omission response time of the group was $59.0 \pm 16.9 \mathrm{~s}$ which was also comparable to that measured previously and much shorter than the interval between rewarded responses from the same sessions (i.e., $271.0 \pm 53.9$ s). More importantly, adding a programmed delay between the response and the drug infusion increased the omission response time by a duration that was close, though not identical, to the added delay (i.e., $+14.5 \mathrm{~s}$ and $+18.3 \mathrm{~s}$ for the 10 -s and 20 -s programmed delays, respectively) $(F 2,30=8.59, p<0.01$, post-hoc Tukey HSD $p$ values $<0.01)$ (Fig. 3b). This latter result suggests that when no programmed delay is present, the length of the omission response time should be close to the underlying delay to cocaine reward. Finally and as expected, adding a programmed delay did not affect the mean intervals between rewarded responses during testing $(F 2,30=0.13, \mathrm{NS})$.

\section{Preference reversal experiment}

As expected, when offered a choice between cocaine and sweet water, rats rapidly developed a strong preference for the latter $(\mathrm{F} 11,231=7.91, p<0.01)$. This preference was manifest from session 2 onward (different from the indifference level of $50 \%$ : $t$ test, d.f. $=21, t$-values $<-2.24, p$ values $<0.05$ ) (Fig. $4 a$ ). On the final three sessions, the large majority of individual rats showed either a marked preference for sweet water $(n=19, \%$ cocaine choice $<40 \%)$ or were indifferent $(n=3, \%$ cocaine choice between $40 \%$ and $60 \%$ ) (Fig. 4b). No individual rat showed a preference for cocaine in this study (i.e., \% cocaine choice $>60 \%$ ). In addition, all rats made their choice relatively rapidly (i.e., $16.5 \pm$ $6.1 \mathrm{~s}$ ), indicating little hesitation. Finally, the amount of sweet reward consumed per sweet water choice was maximal for all individuals (i.e., $0.32 \pm 0.00 \mathrm{ml}$ ).

When a programmed delay was added to both options, rats became slower to make their choice and sometimes did not make a choice within the 5 -min imparted time $(F 4,84=16.76, p<0.01)$ (Fig. S6A). As the result, they completed less choice trials ( $\mathrm{F} 4,84=$ $12.84, p<0.01$ ) (Fig. S6B). This outcome was expectable because adding a programmed delay to the two options should decrease both their values, thereby decreasing the choice stake. In three rats, the \% of completed trials dropped below 25\% when the delay was $40 \mathrm{~s}$ or higher, precluding any reliable analysis of their choice behavior at these programmed delays (Fig. S6C). These rats were thus excluded, leaving a total of 19 rats for final analysis. Overall, increasing the length of the programmed delay caused rats to increase their choice of cocaine and eventually to reverse their preference for sweet water to cocaine $(F 4,72=39.16, p<$ 0.01 ) (Fig. 5a). Preference for cocaine tended to level off above 40 
L Canchy et al.
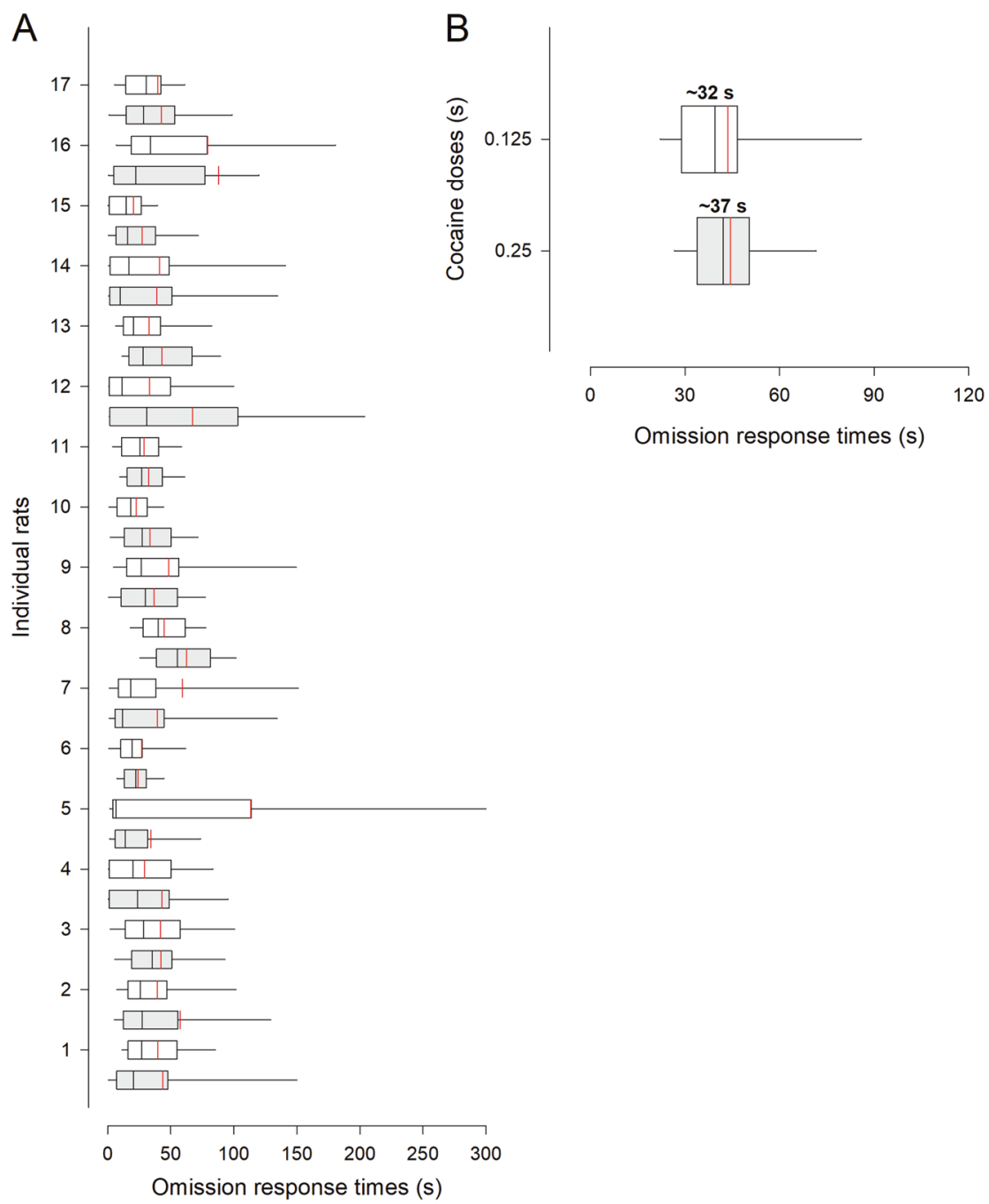

Fig. 2 Omission response times during intravenous cocaine self-administration. a Box plots of omission response times for each individual $(n=17)$ at doses $0.25 \mathrm{mg}$ (light gray) and $0.125 \mathrm{mg}$ (white). Boxes represent the first, second (i.e., median) and third quartiles; vertical red lines within boxes represent the means; error bars represent the 5 and 95 percentiles. $\mathbf{b}$ Box plots of all individual mean omission response times at doses $0.25 \mathrm{mg}$ and $0.125 \mathrm{mg}$. These two doses are represented by two different shadings in the figure. The corresponding statistically indistinguishable shortest delays are indicated above each box.

$s$ and reached statistical significance at the longest delay of $60 \mathrm{~s}$ (above the indifference level of 50\%: $p<0.05, t$-test). Importantly, this effect was not incidental to a reduction in the amount of sweet reward consumed per sweet water choice $(F 4,72=2.24, N S)$ which remained close to the maximum available even at the longest delay of $60 \mathrm{~s}$ (i.e., $0.30 \pm 0.01 \mathrm{ml}$ ).

Interestingly, response latencies during sampling trials were generally congruent with and even predictive of subsequent choice behavior. Consistent with previous research [11], when no programmed delay was added (i.e., during baseline preference for sweet water), rats responded faster for sweet water, the preferred option, than for cocaine $(2.79 \pm 0.74$ versus $9.32 \pm 1.52 ; \mathrm{F} 1,18=17.63, p<$ 0.01 ). When a programmed delay was added to both rewards, rats became slower to respond for either reward, but the increase in response latency was steeper for sweet water than for cocaine $(F 4,72=7.72, p<0.01)$. At the longest programmed delay, rats now responded faster for cocaine, the now preferred reward, than for sweet water $(73.52 \pm 19.70$ versus $123.36 \pm 24.55 ; \mathrm{F} 1,18=8.30, p<$ $0.01)$. Once again, this effect was not incidental to a reduction in the amount of sweet reward consumed per sweet water sampling $(\mathrm{F} 4,72=1.98$, NS) which remained close to the maximum available even at the longest delay of $60 \mathrm{~s}$ (i.e., $0.30 \pm 0.01 \mathrm{ml}$ ).
Though all rats increased their cocaine choice with increased programmed delays, 13 rats reversed their preference to cocaine at $60 \mathrm{~s}$ (i.e., \% cocaine choice $>60 \%$ ) while the other 6 rats either became indifferent $(n=4)$ or continued to slightly prefer sweet water $(n=2)$ (Fig. 5b). Importantly, when we looked at each of these subgroups separately, we found that the majority of rats that reversed their preference to cocaine continued to increase their cocaine choices with increased delays while the other rats did not (Subgroup: $F 4,68=3.37, p<0.05$ ). In the latter rats, cocaine choices tended instead to level off around the indifference level at $40 \mathrm{~s}$. It is thus unlikely that they would have reversed their preference to cocaine at longer programmed delays (Fig. 5c). The levelling-off of their cocaine choices around indifference may suggest that for these few rats, cocaine is not larger, but similar in reward magnitude than sweet water. There were no other differences between these two subgroups during choice testing. They initially acquired the same preference for sweet water and at the same rate than the majority of rats that subsequently reversed their preference to cocaine (Subgroup: F1,17 $=0.01$, NS; Subgroup $x$ Delay: $F 4,68=0.50, N S)$. In addition, regardless of the programmed delays, rats that did not fully reverse their preference completed as many choice trials as rats that fully reversed their 
A

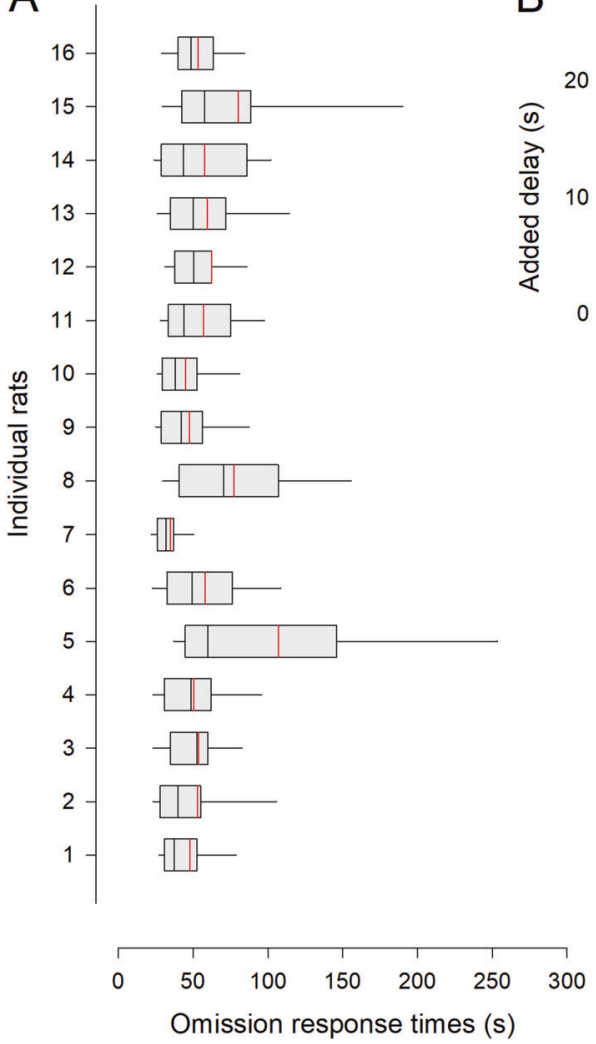

B

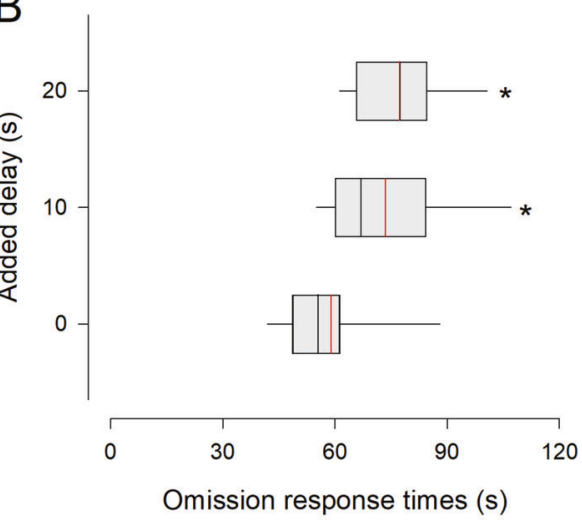

Fig. 3 Effects of adding a programmed drug delay on omission response times. a Box plots of omission response times for each individual $(n=16)$ at the control 0-s delay. Boxes represent the first, second (i.e., median) and third quartiles; vertical red lines within boxes represent the means; error bars represent the 5 and 95 percentiles. $\mathbf{b}$ Box plots of all individual mean omission response times as a function of the added delay. ${ }^{*}$ different from added 0 -s delay, post-hoc Tukey $p$ value $<0.05$.

preference (Subgroup: F1,17 $=0.84$; Subgroup $\times$ Delay: F4,68= 2.40 , NS). They were also equally fast to make their choice (Subgroup: F1,17 = 0.94, NS; Subgroup x Delay: F4,68 = 2.34, NS) and consumed the same amount of sweet reward when they happened to choose this reward (Subgroup: $F 1,17=0.79$, NS; Subgroup x Delay: F4,68=0.64, NS). Finally, when we looked at behavior before choice testing, we found that rats that did not fully reverse their preference to cocaine tended to take less cocaine than the other rats (i.e., total intake: $134.9 \pm 10.0$ versus $179.6 \pm 17.0 \mathrm{mg}$ ), but this difference was not statistically significant $(\mathrm{F} 1,17=2.90$, NS). There was no difference in total sweet intake before choice testing $(24.2 \pm 0.4$ versus $24.0 \pm 0.6 \mathrm{ml})$.

\section{DISCUSSION}

A systematic analysis of the literature revealed that the delays of action of intravenous cocaine on ventral striatal dopamine are of the order of tens of seconds, as was suggested previously [30], and are considerably longer than those measured in response to food reward (see Fig. S3). Overall, our behavioral data confirmed that the delay to cocaine reward is likely tens of seconds long, though data heterogeneity, both between- and within-subject, precluded any inference of a definite delay. Having obtained sufficient converging evidence that cocaine is likely a long-delayed reward, we conducted a preference reversal experiment in rats that were choosing between cocaine and sweet water. Adding a programmed delay to these two options caused all rats to increase their choice of cocaine and a large majority of them to shift their preference from sweet water to cocaine. Taken together, this research confirms the delayed drug reward hypothesis $[8,9]$ and offers a reconciliation of previous cocaine choice studies in rats and the dopamine hypothesis of addiction [1-7]. Thus, for most rats, cocaine reward is supranormal in magnitude, but its inherently longer delay makes it a less desirable option during choice in comparison to smaller, sooner nondrug rewards. Since the delay to cocaine reward is imposed by pharmacokinetics, this also suggests that during choice, pharmacokinetics can trump pharmacodynamics, a conclusion that should be taken more into account in future research, notably when comparing drug versus nondrug rewards.

Thus, previous drug choice studies in rats were likely intertemporal choice studies between a delayed drug reward and an immediate nondrug alternative. A major difference with more classic intertemporal choice studies, however, is that the delay to cocaine reward is less accessible and modifiable than the delay of a typical larger, longer nondrug reward is. As explained above, we have evidence that the delay to cocaine reward is relatively long, but we do not know its exact length, mainly because our available data are indirect and relatively heterogeneous across studies and individuals. We can prolong the delay to cocaine reward by retarding a drug infusion, but we cannot shorten it, at least not sufficiently to make it as immediate as a nondrug reward alternative can be. For instance, in a previous study, we reduced the duration of intravenous cocaine infusion from $4 \mathrm{~s}$ (which is the duration of infusion used in our drug choice studies, including the present study) to $1 \mathrm{~s}$, but this had no observable impact on drug choice outcomes (unpublished findings). This negative finding was not unexpected, however, since the duration of infusion should have little effect on the time course of drug concentration in the body and the brain, and thus on the delay to cocaine reward, except, of course, when the infusion duration is excessively long [28, 64]. Thus, it is not possible to offer rats a drug reward with either a known delay or 


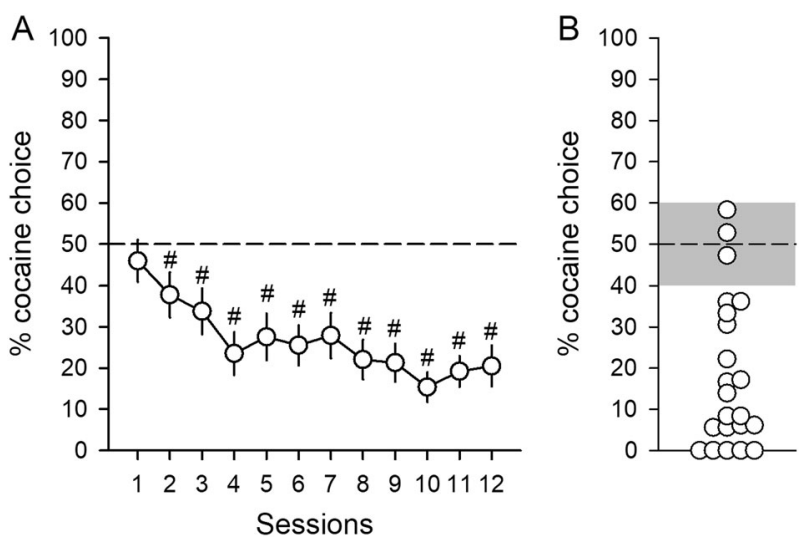

Fig. 4 Preference for sucrose over cocaine during choice. a Mean ( \pm s.e.m.) percentage of cocaine choice across sessions. The horizontal dotted line represents indifference level. \# different from indifference, $t$-test $p$ values $<0.05$. b Average individual preferences over the last three choice sessions. Each point represents a different individual $(n=22)$. The gray zone represents $\pm 10 \%$ around the indifference level.
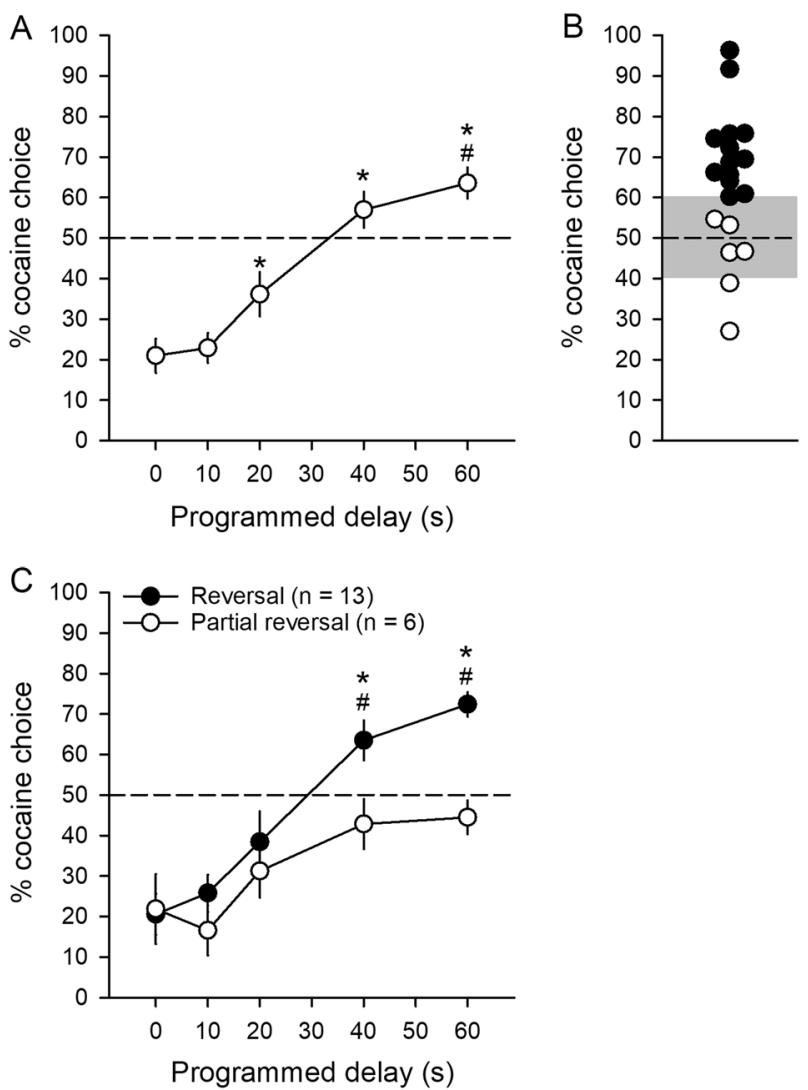

Fig. 5 Effects of adding an increasing programmed delay to both options on preference. a Mean ( \pm s.e.m.) \% cocaine choices as a function of programmed delays. b Average individual preferences over the last three choice sessions with the longest programmed delay of $60 \mathrm{~s}$. Each point represents a different individual $(n=19)$. The gray zone represents $\pm 10 \%$ around the indifference level. c Mean ( \pm s.e.m.) \% cocaine choices in the two subgroups of rats as a function of programmed delays. \# above indifference, $t$-test $p$ values $<0.05 ; *$ different from the other subgroup, post-hoc Tukey $p$ value $<0.05$. no delay at all, a limitation that considerably restricts our ability to compare drug rewards with nondrug rewards. This analysis identifies an unforeseen gap in our knowledge on drug reward that needs to be filled by new research.

According to the delayed drug reward hypothesis, if one were able to offer cocaine reward without a delay, all rats should choose cocaine and this regardless of the magnitude of the immediate nondrug option available. One way to resolve this problem could be to train rats to self-administer cocaine directly into the ventral striatum via chronic intracerebral cannula [65]. However, there is little certitude that the rewarding effects of intrastriatal cocaine will reproduce appropriately the rewarding effects of intravenous cocaine which also increases dopamine levels in several other reward-related brain regions. This factor may explain, at least in part, why intra-striatal cocaine was found in previous research to support only weakly self-administration in rats [66-68]. Thus, if rats were offered a choice between intravenous cocaine and intrastriatal cocaine, it is not sure that they would prefer the intracranial route. This analysis nevertheless raises a number of theoretical issues worth pursuing in future research.

There is, however, an alternative approach that may reproduce the rewarding effects of intravenous cocaine reward perhaps more accurately and equally rapidly than direct intracerebral infusions of cocaine. It consists of using optogenetics to photostimulate selectively and quasi-immediately midbrain dopamine neurons $[69,70]$, thereby increasing dopamine levels in several brain regions, like intravenous cocaine does but more rapidly. Using this approach, it has recently been found that photostimulation of midbrain dopamine neurons induces more individual resistance to foot shock punishment - a measure of addiction-like behavior [71-73] - than intravenous cocaine in mice, suggesting a greater addiction potential $[69,70]$. Specifically, a large majority of mice (i.e., 70\%) developed punishment resistance to photostimulation of midbrain dopamine neurons while only a minority (i.e., less than $20 \%$ ) developed this behavior to intravenous cocaine [69]. Thus, when the effects of cocaine on brain dopamine levels are reproduced but without the long delays, more animals develop addiction-like behavior, as predicted by the delayed drug reward hypothesis $[8,9]$. We predict that if mice were offered a concurrent choice between photostimulation of midbrain dopamine neurons and a sweet reward, they would choose the dopamine neuron stimulation. This hypothesis also predicts that if given a choice, mice should choose photostimulation of midbrain dopamine neurons over intravenous cocaine itself - a prediction that remains to be tested. Importantly, this interpretation does not exclude other possible explanations. For instance, the lower addictive effects of cocaine in comparison to photostimulation of midbrain dopamine neurons may also be due to its action on other neurotransmitters in the brain that could mitigate its action on dopamine [74].

As explained in the Introduction, the delayed drug reward hypothesis was initially developed to explain why rats prefer nondrug reward over intravenous drug reward and reconcile this finding with the dopamine hypothesis of addiction. It is not clear, however, how this hypothesis can also explain the behavior of cocaine-preferring rats that are sometimes observed, though not always (e.g., present study), in drug choice studies [75, 76]. For instance, when we pooled all our cocaine choice experiments, we found that the prevalence of cocaine-preferring rats was about $10 \%$ [10]. This prevalence did not increase significantly after extended access to and escalation of cocaine self-administration [10], suggesting that cocaine preference may be a preexisting individual trait. This interpretation is consistent with subsequent research on the neuronal correlates that predict individual drug preferences in rats [77]. What distinguishes drug-preferring rats from the majority of nondrug-preferring ones remains currently 
poorly understood, however. We previously suggested that these individuals may be homologous to the minority of cocaine users who go on to develop addiction in humans $[75,76]$. However, the delayed drug reward hypothesis suggests another, more counterintuitive possibility. Cocaine-preferring rats could simply be less sensitive to long reward delays compared to the majority of rats. If true, they should also behave less impulsively in other intertemporal choice settings, choosing more often the larger, more delayed options than the other rats. In other words, low choice impulsivity would predispose to greater drug choice in rats. Obviously, this is the opposite of what we know about the relationship between choice impulsivity and vulnerability to drug use and addiction in people [78-81]. People who behave less impulsively in intertemporal choice settings are less, not more, vulnerable to use drugs and go on to develop addiction. Clearly, further research is needed to resolve this apparent translational gap between rats and humans (for additional discussion of this translational gap, see [8, 82]).

Finally, previous research has shown that after prior experience with cocaine self-administration, cocaine can also induce a conditioned increase in nucleus accumbens dopamine levels via interoceptive sensory inputs from the periphery [30, 83]. Though this conditioned activation is lower in magnitude than that produced by the direct action of cocaine on brain dopamine uptake, it is thought to be faster. However, we do not know how much faster it is. No study has so far directly measured the delay of onset of this conditioned activation with the required temporal resolution. In fact, even it was near immediate, it would not be sufficient to bias choice towards cocaine, as it was consistently observed in previous drug choice studies [8]. Given a choice between two immediate cues, rats prefer the cue that predicts a small, immediate reward over the cue that predicts a larger, more delayed reward. As expected, this preference is correlated with the ability of each conditioned cue to induce dopamine levels in the nucleus accumbens [84]. Thus, the immediacy of a conditioned cue does not compensate for the delay of the predicted reward.

\section{CONCLUSIONS}

In conclusion, the present study provides strong evidence for the delayed drug reward hypothesis and offers a reconciliation of cocaine choice studies in rats and the dopamine hypothesis of addiction. Thus, for most rats, cocaine reward is supranormal in magnitude, but its inherently long delay makes it a less preferred desirable option during choice in comparison to nondrug rewards. Since the delay to cocaine reward is imposed by pharmacokinetics, this suggests that pharmacokinetics can trump pharmacodynamics in drug self-administration studies, a conclusion that should be taken more into account in future research, notably when comparing drug versus nondrug rewards. Finally, this study also reveals several important knowledge gaps, notably in our understanding of drug reward delays, that need to be filled by future experimental and theoretical research.

\section{FUNDING AND DISCLOSURE}

This work was supported by the French Research Council (CNRS), the Université de Bordeaux, the Ministère de l'Enseignement Supérieur et de la Recherche (MESR) and the Fondation pour la Recherche Médicale (FRM DPA20140629788). The authors declare no competing interests.

\section{ACKNOWLEDGEMENTS}

We thank Christophe Bernard, Mathieu Louvet and Eric Wattelet for administrative assistance. We also thank Drs. Karine Guillem, Magalie Lenoir, Hanna Pickard and Youna Vandaele for their helpful comments on a previous version of the manuscript. Finally, we would like to thank the reviewers for their constructive criticisms.

\section{AUTHOR CONTRIBUTIONS}

SHA conceived the work. SHA, LC, PG contributed to the design of the experiments. LC and SHA performed the systematic analyses of the literature. LC, PG, AD, CVM carried out the experiments. LC, PG, AD collected the experimental data. SHA, LC analyzed the data. SHA wrote the paper. All authors reviewed content and approved the final version of the manuscript.

\section{ADDITIONAL INFORMATION}

Supplementary Information accompanies this paper at (https://doi.org/10.1038/ s41386-020-0786-9).

Publisher's note Springer Nature remains neutral with regard to jurisdictional claims in published maps and institutional affiliations.

\section{REFERENCES}

1. Di Chiara G. Drug addiction as dopamine-dependent associative learning disorder. Eur J Pharm. 1999;375:13-30.

2. Keiflin R, Janak PH. Dopamine prediction errors in reward learning and addiction: from theory to neural circuitry. Neuron. 2015;88:247-63.

3. Wise RA. Dopamine, learning and motivation. Nat Rev Neurosci. 2004;5:483-94.

4. Wise RA, Koob GF. The development and maintenance of drug addiction. Neuropsychopharmacology. 2014;39:254-62.

5. Robinson TE, Berridge KC. Review. The incentive sensitization theory of addiction some current issues. Philos Trans R Soc Lond B Biol Sci. 2008;363:3137-46.

6. Redish AD. Addiction as a computational process gone awry. Science. 2004; 306:1944-7.

7. Kalivas PW, Volkow ND. The neural basis of addiction: a pathology of motivation and choice. Am J Psychiatry. 2005;162:1403-13.

8. Ahmed SH. Trying to make sense of rodents' drug choice behavior. Prog Neuropsychopharmacol Biol Psychiatry. 2018;87(Pt A):3-10.

9. Ahmed SH. Individual decision-making in the causal pathway to addiction: contributions and limitations of rodent models. Pharm Biochem Behav. 2018;164:22-31.

10. Cantin L, Lenoir M, Augier E, Vanhille N, Dubreucq S, Serre F, et al. Cocaine is low on the value ladder of rats: possible evidence for resilience to addiction. PLoS One. 2010;5:e11592.

11. Lenoir M, Serre F, Cantin L, Ahmed SH. Intense sweetness surpasses cocaine reward. PLoS One. 2007;2:e698.

12. Madsen HB, Ahmed SH. Drug versus sweet reward: greater attraction to and preference for sweet versus drug cues. Addict Biol. 2015;20:433-44.

13. Bagley JR, Adams J, Bozadjian RV, Bubalo L, Ploense KL, Kippin TE. Estradiol increases choice of cocaine over food in male rats. Physiol Behav. 2019;203:18-24.

14. Kearns DN, Kim JS, Tunstall BJ, Silberberg A. Essential values of cocaine and nondrug alternatives predict the choice between them. Addict Biol. 2017;22:1501-14.

15. Tunstall BJ, Kearns DN. Reinstatement in a cocaine versus food choice situation: reversal of preference between drug and non-drug rewards. Addict Biol. 2014; 19:838-48.

16. Tunstall BJ, Kearns DN. Sign-tracking predicts increased choice of cocaine over food in rats. Behav Brain Res. 2015;281:222-8.

17. Tunstall BJ, Kearns DN. Cocaine can generate a stronger conditioned reinforcer than food despite being a weaker primary reinforcer. Addict Biol. 2016;21:282-93.

18. Tunstall BJ, Riley AL, Kearns DN. Drug specificity in drug versus food choice in male rats. Exp Clin Psychopharmacol. 2014;22:364-72.

19. Caprioli D, Venniro M, Zeric T, Li X, Adhikary S, Madangopal R, et al. Effect of the novel positive allosteric modulator of metabotropic glutamate receptor 2 AZD8529 on incubation of methamphetamine craving after prolonged voluntary abstinence in a rat model. Biol Psychiatry. 2015;78:463-73.

20. Caprioli D, Zeric T, Thorndike EB, Venniro M. Persistent palatable food preference in rats with a history of limited and extended access to methamphetamine selfadministration. Addict Biol. 2015;20:913-26.

21. Venniro $M$, Caprioli $D$, Zhang $M$, Whitaker LR, Zhang $S$, Warren $B L$, et al. The anterior insular cortex->central amygdala glutamatergic pathway is critical to relapse after contingency management. Neuron. 2017;96:414-27. e8

22. Lenoir M, Cantin L, Vanhille N, Serre F, Ahmed SH. Extended heroin access increases heroin choices over a potent nondrug alternative. Neuropsychopharmacology. 2013;38:1209-20.

23. Schwartz LP, Kim JS, Silberberg A, Kearns DN. Heroin and saccharin demand and preference in rats. Drug Alcohol Depend. 2017;178:87-93.

24. Vandaele Y, Vouillac-Mendoza C, Ahmed SH. Inflexible habitual decision-making during choice between cocaine and a nondrug alternative. Transl Psychiatry. 2019;9:109.

25. Venniro $M$, Russell Tl, Zhang M, Shaham Y. Operant social reward decreases incubation of heroin craving in male and female rats. Biol Psychiatry. 2019;86:848-56. 
26. Venniro M, Zhang M, Caprioli D, Hoots JK, Golden SA, Heins C, et al. Volitional social interaction prevents drug addiction in rat models. Nat Neurosci. 2018;21:1520-29.

27. Welling PG. Pharmacokinetics: processes and mathematics. Washington, DC: American Chemical Society; 1986.

28. Pan HT, Menacherry S, Justice JB Jr. Differences in the pharmacokinetics of cocaine in naive and cocaine-experienced rats. J Neurochemistry. 1991;56:1299-306.

29. Lau CE, Sun $L$. The pharmacokinetic determinants of the frequency and pattern of intravenous cocaine self-administration in rats by pharmacokinetic modeling. Drug Metab Disposition. 2002;30:254-61.

30. Wise RA, Kiyatkin EA. Differentiating the rapid actions of cocaine. Nat Rev Neurosci. 2011;12:479-84.

31. Ainslie G. Specious reward: a behavioral theory of impulsiveness and impulse control. Psychol Bull. 1975;82:463-96.

32. Mazur JE. Choice, delay, probability, and conditioned reinforcement. Anim Learn Behav. 1997:25:131-47.

33. Stevens JE, Stephens DW. The adaptive nature of impulsivity. In: Madden GJ, Bickel WK, editors. Impulsivity: the behavioral and neurological science of discounting. Washington, DC: American Psychological Association; 2010. p. 361-88.

34. Vanderveldt A, Oliveira L, Green L. Delay discounting: pigeon, rat, human - does it matter? J Exp Psychol: Anim Learn cognition. 2016;42:141-62.

35. van Gaalen MM, van Koten R, Schoffelmeer ANM, Vanderschuren LMJ. Critical involvement of dopaminergic neurotransmission in impulsive decision making. Biol Psychiatry. 2006;60:66-73.

36. Evenden JL, Ryan CN. The pharmacology of impulsive behaviour in rats: the effects of drugs on response choice with varying delays of reinforcement. Psychopharmacol (Berl). 1996;128:161-70.

37. Richards JB, Mitchell SH, De Wit H, Seiden LS. Determination of discount functions in rats with an adjusting-amount procedure. J Exp Anal Behav. 1997;67:353-66.

38. Logan FA. Decision making by rats: delay versus amount of reward. J Comp Physiol Psychol. 1965;59:1-12.

39. Bradshaw CM, Szabadi E. Choice between delayed reinforcers in a discrete-trials schedule: the effect of deprivation level. Quaterly J Exp Psychol. 1992;44B:1-16.

40. Cardinal RN, Robbins TW, Everitt BJ. The effects of d-amphetamine, chlordiazepoxide, alpha-flupenthixol and behavioural manipulations on choice of signalled and unsignalled delayed reinforcement in rats. Psychopharmacol (Berl). 2000;152:362-75.

41. Peterson JR, Hill CC, Kirkpatrick K. Measurement of imulsive choice in rats: sameand alternate-form test-retest reliability and temporal tracking. J Exp Anal Behav. 2015;103:166-79.

42. Panlilio LV, Secci ME, Schindler CW, Bradberry CW. Choice between delayed food and immediate opioids in rats: treatment effects and individual differences. Psychopharmacol (Berl). 2017;234:3361-73.

43. Secci ME, Factor JA, Schindler CW, Panlilio LV. Choice between delayed food and immediate oxycodone in rats. Psychopharmacol (Berl). 2016;233:3977-89.

44. Wise RA, Kiyatkin EA. On the speed of cocaine. Nat Rev Neurosci. 2011;12:700.

45. Aragona BJ. The regional specificity of rapid actions of cocaine. Nat Rev Neurosci. 2011;12:700.

46. Green L, Estle SJ. Preference reversals with food and water reinforcers in rats. $J$ Exp Anal Behav. 2003;79:233-42.

47. Ainslie GW, Herrnstein RJ. Preference reversal and delayed reinforcement. Anim Learn Behav. 1981;9:476-82.

48. Green L, Fisher EBJ, Perlow S, Sherman L. Preference reversal and self-control: choice as a function of reward amount and delay. Behav Anal Lett. 1981;1:43-51.

49. Beeby $E$, White KG. Preference reversal between impulsive and self-control choice. J Exp Anal Behav. 2013;99:260-76.

50. Krebs CA, Anderson KG. Preference reversals and effects of d-amphetamine on delay discounting in rats. Behavioural Pharmacol. 2012;23:228-40.

51. Ito M, Asaki K. Choice behavior of rats in a concurrent-chains schedule: amount and delay of reinforcement. J Exp Anal Behav. 1982;37:383-92.

52. Kobayashi S, Schultz W. Influence of reward delays on responses of dopamine neurons. J Neurosci. 2008;28:7837-46.

53. Green L, Myerson J. A discounting framework for choice with delayed and probabilistic rewards. Psychol Bull. 2004;130:769-92.

54. Kirby KN, Herrnstein RJ. Preference reversals due to myopic discounting of delayed reward. Psychological Sci. 1995;6:83-89.

55. Sozou PD. On hyperbolic discounting and uncertain hazard rates. Proc R Soc B. 1998;265:2015-20.

56. Blanchard TC, Pearson JM, Hayden BY. Postreward delays and systematic biases in measures of animal temporal discounting. Proc Natl Acad Sci USA. 2013;110:15491-96.

57. Volkow ND, Michaelides $M$, Baler R. The neuroscience of drug reward and addiction. Physiol Rev 2019;99:2115-40.
58. Pickens R, Meisch RA, Thompson T. Drug self-administration: an analysis of the reinfrocing effects of drugs. In: Iversen $\mathrm{LL}$, Iversen SD, Solomon $\mathrm{SH}$, editor. Handbook of psychopharmacology: drugs of abuse. New York: Plenum Press; 1978. p. 1-37.

59. Yokel RA. Intravenous self-administration: response rates, the effects of pharmacological challenges, and drug preference. In: Bozarth MA, editor. Methods of assessing the reinforcing properties of abused drugs. New York: Springer-Verlag; 1987. p. 1-33.

60. Wise RA. Intravenous drug self-administration: a special case of positive reinforcement. In: Bozarth MA, editor. Methods of assessing the reinforcing properties of abused drugs. New York: Springer-Verlag; 1987. p. 117-41.

61. Ahmed SH, Koob GF. Transition to drug addiction: a negative reinforcement model based on an allostatic decrease in reward function. Psychopharmacol (Berl). 2005;180:473-90.

62. Vandaele Y, Cantin L, Serre F, Vouillac-Mendoza C, Ahmed SH. Choosing under the influence: a drug-specific mechanism by which the setting controls drug choices in rats. Neuropsychopharmacology. 2016;41:646-57.

63. Freese L, Durand A, Guillem K, Ahmed SH. Pre-trial cocaine biases choice toward cocaine through suppression of the nondrug option. Pharm Biochem Behav. 2018;173:65-73.

64. Minogianis EA, Shams WM, Mabrouk OS, Wong J-MT, Brake WG, Kennedy RT, et al. Varying the rate of intravenous cocaine infusion influences the temporal dynamics of both drug and dopamine concentrations in the striatum. Eur J Neurosci. 2019;50:2054-64.

65. Goeders NE, Smith JE. Intracranial self-administration methodologies. Neurosci Biobehav Rev. 1987;11:319-29.

66. Carlezon WA, Devine DP, Wise RA. Habit-forming actions of nomifensine in nucleus accumbens. Psychopharmacol (Berl). 1995;122:194-97.

67. Goeders NE, Smith JE. Cortical dopaminergic involvement in cocaine reinforcement. Science 1983;221:773-75.

68. Rodd-Henricks ZA, McKenzie DL, Li TK, Murphy JM, McBride WJ. Cocaine is selfadministered in the shell but not the core of the nucleus accumbens of Wistar rats. J Pharmacol Exp Therapeutics. 2002;303:1216-26.

69. Pascoli V, Terrier J, Hiver A, Luscher C. Sufficiency of mesolimbic dopamine neuron stimulation for the progression to addiction. Neuron. 2015;88:1054-66.

70. Pascoli V, Hiver A, Van Zessen R, Loureiro M, Achargui R, Harada M, et al. Stochastic synaptic plasticity underlying compulsion in a model of addiction. Nature. 2018;564:366-71.

71. Deroche-Gamonet V, Belin D, Piazza PV. Evidence for addiction-like behavior in the rat. Science. 2004;305:1014-7.

72. Chen BT, Yau HJ, Hatch C, Kusumoto-Yoshida I, Cho SL, Hopf FW, et al. Rescuing cocaine-induced prefrontal cortex hypoactivity prevents compulsive cocaine seeking. Nature. 2013;496:359-62.

73. Pelloux Y, Everitt BJ, Dickinson A. Compulsive drug seeking by rats under punishment: effects of drug taking history. Psychopharmacol (Berl). 2007;194:127-37.

74. Lüscher C, Robbins TW, Everitt BJ. The transition to compulsion in addiction. Nat Rev Neurosci. 2020;21:247-63.

75. Ahmed $\mathrm{SH}$, Lenoir $\mathrm{M}$, Guillem K. Neurobiology of addiction versus drug use driven by lack of choice. Curr Opin Neurobiol. 2013;23:581-7.

76. Ahmed SH. Validation crisis in animal models of drug addiction: beyond nondisordered drug use toward drug addiction. Neurosci Biobehav Rev. 2010;35:172-84.

77. Guillem K, Ahmed SH. Preference for cocaine is represented in the orbitofrontal cortex by an increased proportion of cocaine use-coding neurons. Cereb Cortex. 2018;28:819-32.

78. Volkow ND, Baler RD. NOW vs LATER brain circuits: implications for obesity and addiction. Trends Neurosci. 2015;38:345-52.

79. Bickel WK, Koffarnus MN, Moody L, Wilson AG. The behavioral- and neuroeconomic process of temporal discounting: a candidate behavioral marker of addiction. Neuropharmacology. 2014;76(Pt B):518-27.

80. de Wit H. Impulsivity as a determinant and consequence of drug use: a review of underlying processes. Addict Biol. 2009;14:22-31.

81. Rung JM, Peck S, Hinnenkamp JE, Preston E, Madden GJ. Chaging delay discounting and impulsive choice: implications for addictions, prevention, and human health. Perspect Behav Sci. 2019;42:397.

82. Ahmed SH. "A walk on the wild side" of addiction: the history and significance of animal models. In: Pickard $\mathrm{H}$, Ahmed SH, editors. The Routledge handbook of philosophy and science of addiction. New York: Routledge; 2019. p. 192-204.

83. Wise RA, Wang B, You ZB. Cocaine serves as a peripheral interoceptive conditioned stimulus for central glutamate and dopamine release. PLoS One. 2008;3:e2846.

84. Saddoris MP, Sugam JA, Stuber GD, Witten IB, Deisseroth K, Carelli RM. Mesolimbic dopamine dynamically tracks, and is causally linked to, discrete aspects of value-based decision making. Biol Psychiatry. 2015;77:903-11. 